\title{
Steffens, Karin
}

\section{Menschenrechte und schulische Bildung im Labyrinth der deutschen}

\section{Einwanderungspolitik}

Steffens, Gerd [Hrsg.]; Weiß, Edgar [Hrsg.]: Menschenrechte und Bildung. Frankfurt, Main : Lang 2011, S. 271-285. - (Jahrbuch für Pädagogik; 2011)

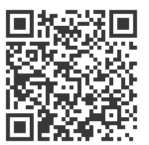

Quellenangabe/ Reference:

Steffens, Karin: Menschenrechte und schulische Bildung im Labyrinth der deutschen

Einwanderungspolitik - In: Steffens, Gerd [Hrsg.]; Weiß, Edgar [Hrsg.]: Menschenrechte und Bildung.

Frankfurt, Main : Lang 2011, S. 271-285 - URN: urn:nbn:de:0111-pedocs-128197 - DOI:

$10.25656 / 01: 12819$

https://nbn-resolving.org/urn:nbn:de:0111-pedocs-128197

https://doi.org/10.25656/01:12819

in Kooperation mit / in cooperation with:

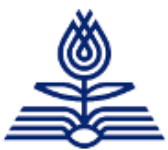

\section{PETER LANG}

INTERNATIONALER VERLAG DER WISSENSCHAFTEN

http://www.peterlang.com

\section{Nutzungsbedingungen}

Gewährt wird ein nicht exklusives, nicht übertragbares, persönliches und beschränktes Recht auf Nutzung dieses Dokuments. Dieses Dokument ist ausschließlich für den persönlichen, nicht-kommerziellen Gebrauch bestimmt. Die Nutzung stellt keine Übertragung des Eigentumsrechts an diesem Dokument dar und gilt vorbehaltlich der folgenden Einschränkungen Auf sämtlichen Kopien dieses Dokuments müssen alle Urheberrechtshinweise und sonstigen Hinweise auf gesetzlichen Schutz beibehalten werden. Sie dürfen dieses Dokument nicht in irgendeiner Weise abändern, noch dürfen Sie dieses Dokument für öffentliche oder kommerzielle Zwecke vervielfältigen, öffentlich ausstellen, aufführen, vertreiben oder anderweitig nutzen.

Mit der Verwendung dieses Dokuments erkennen Sie die Nutzungsbedingungen an.

\section{Terms of use}

We grant a non-exclusive, non-transferable, individual and limited right to using this document.

This document is solely intended for your personal, non-commercial use. Use of this document does not include any transfer of property rights and it is conditional to the following limitations: All of the copies of this documents must retain all copyright information and other information regarding legal protection. You are not allowed to alter this document in any way, to copy it for public or commercial purposes, to exhibit the document in public, to perform, distribute or otherwise use the document in public.

By using this particular document, you accept the above-stated conditions of use.

\section{Kontakt / Contact:}

\section{peDOCS}

DIPF | Leibniz-Institut für Bildungsforschung und Bildungsinformation Informationszentrum (IZ) Bildung

E-Mail: pedocs@dipf.de

Internet: www.pedocs.de

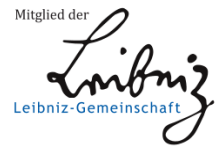




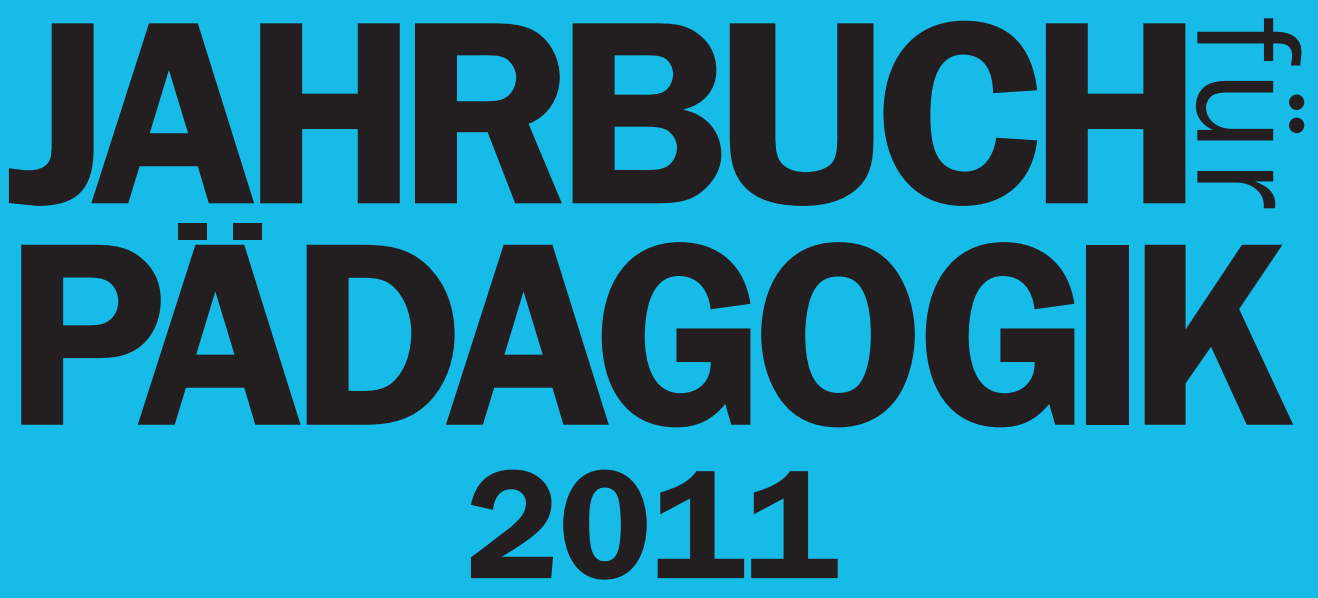

Menschenrechte und Bildung

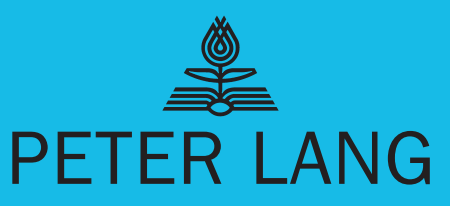


Im Bereich Menschenrechte sind Bildung und Pädagogik heute auf das engste mit den Diskursen verflochten, in denen sich Gesellschaften - und eine sich herausbildende Weltgesellschaft - über sich selbst und ihre Entwicklung verständigen. Die Gegenwartsbedeutung dieses Themenkomplexes für Einwanderungsgesellschaften wird zudem durch die Alltagspräsenz von Fragen rund um Menschen- und Bürgerrechte, deren Verständnis und Handhabung, insbesondere im Bereich der Bildung, unterstrichen. Kaum zu übersehen ist auch, wie dringlich angesichts der Vorherrschaft marktorientierten Denkens die kritische Erneuerung und Stärkung von Denkweisen ist, die Gesellschaft und Bildung auf der Grundlage wechselseitiger Anerkennung gesellschaftlicher Individuen entwerfen wollen, deren Legitimation ihre Menschenrechte und die aller anderen sind.

Gerd Steffens, Prof. Dr. phil., lehrte bis 2007 Politische Bildung und ihre Didaktik an der Universität Kassel.

Edgar Weiß, Dr. phil. habil., ist Vertretungsprofessor für Schulpädagogik an der Universität Siegen. 
Jahrbuch für Pädagogik 2011

Menschenrechte und Bildung 


\title{
JAHRBUCH FÜR PÄDAGOGIK 2011
}

\section{Menschenrechte und Bildung}

\author{
Redaktion: \\ Gerd Steffens und Edgar Weiß
}

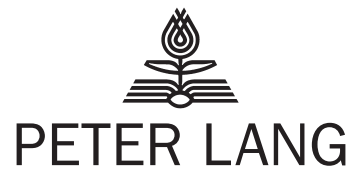

Internationaler Verlag der Wissenschaften 


\section{JAHRBUCH FÜR PÄDAGOGIK}

Begründet von:

Kurt Beutler, Ulla Bracht, Hans-Jochen Gamm, Klaus Himmelstein, Wolfgang Keim, Gernot Koneffke, Karl Christoph Lingelbach, Gerd Radde, Ulrich Wiegmann, Hasko Zimmer

Herausgeberlnnen:

Armin Bernhard (Essen-Duisburg),

Martin Dust (Saarbrücken), Sven Kluge (Essen-Duisburg), Ingrid Lohmann (Hamburg), Andreas Merkens (Hamburg), Johanna Mierendorff (Halle-Wittenberg), Gerd Steffens (Kassel), Edgar Weiß (Siegen)

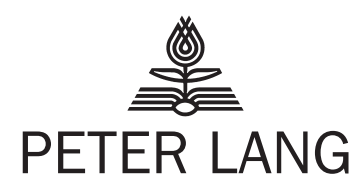

Frankfurt am Main · Berlin · Bern · Bruxelles · New York · Oxford · Wien 


\title{
Bibliografische Information der Deutschen Nationalbibliothek
} Die Deutsche Nationalbibliothek verzeichnet diese Publikation in der Deutschen Nationalbibliografie; detaillierte bibliografische Daten sind im Internet über http://dnb.d-nb.de abrufbar.

\author{
E-ISBN 978-3-653-05157-5 (E-Book) \\ DOI 10.3726/978-3-653-05157-5 \\ ISSN 0941-1461 \\ ISBN 978-3-631-62109-7 \\ (C) Peter Lang GmbH \\ Internationaler Verlag der Wissenschaften \\ Frankfurt am Main 2011 \\ Alle Rechte vorbehalten.
}

Das Werk einschließlich aller seiner Teile ist urheberrechtlich geschützt. Jede Verwertung außerhalb der engen Grenzen des Urheberrechtsgesetzes ist ohne Zustimmung des Verlages unzulässig und strafbar. Das gilt insbesondere für Vervielfältigungen, Übersetzungen, Mikroverfilmungen und die Einspeicherung und Verarbeitung in elektronischen Systemen. 


\section{Karin Steffens}

\section{Menschenrechte und schulische Bildung im Labyrinth der deutschen Einwanderungspolitik}

Migration ist ein Konstituens der menschlichen Gattung und ein Kontinuum in der Menschheitsgeschichte. In ihr sind Wanderungen keine Ausnahmezustände, sondern Normalität. Auch die neuere Geschichte Deutschlands und Europas ist eine Geschichte der Wanderungsbewegungen: zunächst - in den vergangenen Jahrhunderten - nach Übersee, angetrieben meist von Hunger und Not, aber auch von Abenteuerlust und Goldgier oder der Neugier auf die Welt. Seit den letzten Jahrzehnten des 19. Jahrhunderts haben Industrialisierung und Kriege, aber auch die generelle Mobilität von Arbeitskraft so umfassende Bevölkerungsbewegungen in Mitteleuropa ausgelöst, dass heute familiäre Sesshaftigkeit, die sich über mehrere Generationen erstreckt, eine fast verschwindende Ausnahme ist.

Um den in der Industrialisierung sprunghaft ansteigenden Arbeitskräftebedarf zu stillen, hatte man seit den 80er Jahren des 19. Jahrhunderts in Deutschland auf ein Beschaffungsmodell aus der Agrargesellschaft zurückgegriffen. Wegen des naturgemäß saisonalen Charakters der in der Landwirtschaft anfallenden Arbeiten hatten sich hier saisonale Wanderungen eingespielt. Sie wurden mit dem Aufschwung der so genannten Gründerjahre des Deutschen Reichs als zeitlich eingegrenzte „Nutzung“ von Arbeitskräften - damals vor allem aus Polen und Italien systematisiert. Obgleich industrielle Produktion keineswegs mehr saisonal ist, blieb die agrarische Fiktion vom „Saisonarbeiter" bestimmend für ein Wunschbild von Arbeitskraft, die wie aus dem Nichts da ist, wenn man sie braucht, und stillschweigend verschwindet, wenn die Ernte eingebracht ist. Nach diesem Wunschbild noch wurde der "Gastarbeiter" modelliert, als im Aufschwung der Bundesrepublik das interne Arbeitskräftereservoir nicht mehr ausreichte. Auch wenn die „Saison“, für die er angeworben worden war, jetzt als die des konjunkturellen Aufschwungs oder eines vorübergehenden Engpasses an Arbeitskraft gedacht wurde, entscheidend blieb die zeitliche Begrenzung, die Vorstellung, dass die Arbeitskraft ohne Folgekosten verschwand, wenn sie nicht mehr gebraucht wurde - eine ideale Grundlage für eine Praxis des Hin- und Herschiebens der Ware Arbeitskraft, als der große Arbeitskräftebedarf seit den späten fünfziger Jahren in Europa und insbesondere in Deutschland eine starke Sogwirkung auszuüben begann, die durch gezielte Anwerbungskampagnen noch verstärkt wurde.

Diese paradoxe Konstruktion des „Gastarbeiters“ als einer Arbeitskraft, die arbeiten und nur vorübergehend da sein, aber nicht dazu gehören soll, bildet so 
etwas wie das emblematische Leitmotiv einer Mentalität, die Migration als historische Normalität leugnet, die Einheit von Territorium und Ethnie für naturgegeben und unberührbar hält und die die Quelle aller seitherigen und heutigen „Integrationsdebatten" bildet. Vor dem Hintergrund dieser Paradoxien eines kollektiven Geschichts- und Selbstverständnisses wende ich mich im Folgenden zunächst der rechtlichen Konstruktion des „Gastarbeiters“ als „Ausländer“ zu (1), frage danach nach deren Verhältnis zu menschenrechtlichen Bestimmungen bis hin zum Recht auf Bildung (2), um dann an diesem Faden einen Weg durchs Labyrinth der deutschen Bildungspolitik seit den sechziger Jahren zu suchen (3).

\section{Regelungsziel: Anwesend, aber nicht zugehörig}

Als 1955 die ersten „Gastarbeiter“ kamen, wurde ihre Arbeits- und Aufenthaltserlaubnis auf der Grundlage der wieder eingeführten saisonal befristeten Legitimationskarte von 1905 geregelt. In den 60er Jahren, als die Zahl der Arbeitskräfte aus dem Ausland die Millionengrenze überschritt, schien das nicht mehr ausreichend. Nun wurde die Ausländerpolizeiverordnung von 1938, die dann den Einsatz von Fremdarbeitern in der Kriegswirtschaft geregelt hatte, 1965 als Ausländergesetz fast unverändert in Kraft gesetzt. Die Aufenthaltsgenehmigungen waren grundsätzlich auf 1 bis höchstens 2 Jahre befristet. Entscheidendes Kriterium für die Vergabe von Arbeits- und Aufenthaltsgenehmigungen sind in der Ausländergesetzgebung seit 1905 bis zum gegenwärtigen Aufenthaltsgesetz die Bedürfnisse und Interessen des Deutschen Reiches bzw. der Bundesrepublik Deutschland.

Seit dem Anwerbestopp im November 1973 - der das bisher gewollte Kommen und Gehen unmöglich machte - stellte sich das Faktum, dass Deutschland ein Einwanderungsland mit immer hier lebenden Menschen ausländischer Herkunft ist, als gesellschaftliche Realität her, ohne dass während der folgenden Jahrzehnte diesem Tatbestand politisch und gesetzlich Rechnung getragen wurde. Dies geschah erst in Ansätzen mit Beginn des 21. Jahrhunderts, also erst knapp 40 Jahre später mit dem Aufenthaltsgesetz von 2004.

Doch auch nach diesem Gesetz gilt für Menschen, die nicht deutsche Staatsbürger und -bürgerinnen sind, nach wie vor ein grundsätzliches Aufenthaltsverbot mit Erlaubnisvorbehalt. Nach §4, Abs.1 des Aufenthaltsgesetzes (AufenthG) bedürfen Ausländerinnen und Ausländer bereits „für die Einreise und den Aufenthalt im Bundesgebiet eines Aufenthaltstitels“ (AufenthG 2004). Besondere Freizügigkeitsreglungen gelten lediglich für EU-Bürgerinnen und -Bürger und bis zu einem bestimmten Grad auch für Mitglieder von Staaten, die ein Assoziationsabkommen mit der EWG, wie z.B. die Türkei 1963, abgeschlossen haben. 
Ebenso gilt nach $\S 4$ Abs.2 des AufenthG ein Arbeitsverbot mit Erlaubnisvorbehalt, wonach nur „ein Aufenthaltstitel [...] zur Ausübung einer Erwerbstätigkeit [berechtigt], sofern es nach diesem Gesetz bestimmt ist oder der Aufenthaltstitel die Ausübung der Erwerbstätigkeit ausdrücklich erlaubt" (AufenthG 2004). Die Möglichkeit, eine Arbeitserlaubnis zu erhalten, war bis 2010 zusätzlich nach den $\S \S 285$ und 286 SGB VIII, der sogenannten Vorrangigkeitsklausel einer Fortschreibung des sogenannten Inländerprimats von 1921 - erschwert, wonach deutsche Staatsbürger und -bürgerinnen und EU-Bürgerinnen und Bürger sowie Einwanderer und Einwanderinnen aus Staaten außerhalb der EU mit Aufenthaltsberechtigung bzw. Niederlassungserlaubnis, die alle keiner Arbeitserlaubnis bedürfen, vorrangig auf Arbeitsplätze vermittelt werden mussten.

Weiterhin legt das Aufenthaltsgesetz in $\S 5$ fest, dass ,die Erteilung eines Aufenthaltstitels [...] in der Regel voraus[setzt], dass der Lebensunterhalt gesichert ist". Sollte wegen längerer, vor allem unverschuldeter Arbeitslosigkeit die Inanspruchnahme von Sozialhilfe notwendig werden, so kann die Ausländerbehörde nach $\S 55$ Abs. 6 und 7 AufenthG im Ermessen entscheiden, ob ,ein Ausländer [...] ausgewiesen werden [kann]“, wenn er ,für sich, seine Familienangehörigen oder für sonstige Haushaltsangehörige Sozialhilfe in Anspruch nimmt" (Abs. 6) oder „Hilfe zur Erziehung außerhalb der eigenen Familie oder Hilfe für junge Volljährige [...] erhält“ (Abs.7) (AufenthG 2004).

Die gesetzliche Grundlage für das Einwanderungs- und Niederlassungsverbot unter Erlaubnisvorbehalt für sogenannte Drittstaater und Drittstaaterinnen, also für Bürgerinnen und Bürger aus Staaten außerhalb der EU, bildet zugleich die Grundlage für alle weiteren gesetzlichen Regelungen, die Ausgrenzung und Ungleichbehandlung im Einwanderungsland Deutschland nach sich ziehen. Der diese Sondergesetzgebung bestimmende Ausländerstatus enthält den Zugewanderten, Gebliebenen und hier Geborenen mit ausländischem Pass nämlich große Teile derjenigen Rechte vor, die grundgesetzlich und menschenrechtlich allen Menschen ohne Ansehen der Herkunft, des Geschlechts, von Religion und Kultur gleichermaßen angeboren sind und ihnen unveräußerlich als Garantie ihrer Menschenwürde und Freiheits- und Gleichheitsrechte zustehen."Die Nicht-Staatsangehörigkeit ist in entscheidender Weise auch eine Nicht-Zugehörigkeit und Ausschließung, die sich durch einen expliziten Ausländerstatus definiert", stellt Heckmann (1992, S.238) zurecht fest. Hilal Sezgin (2011, S.50) betont in diesem Zusammenhang: „Denn tatsächlich geht es um Anerkennung und um das Vorenthalten derselben. Nicht nur um Anerkennung für eine einzelne Leistung, sondern um die Anerkennung als Subjekt eines eigenen Lebens“.

In diese Überlegungen mit einzubeziehen ist, wie ich meine, dass der Status der Nicht-Zugehörigkeit nicht nur zu Ausschließung und Nichtanerkennung führt, sondern bei gleichzeitig massiv von den Eingewanderten eingeforderten Integra- 
tionsleistungen zugleich ein schier unüberwindliches Integrationshindernis darstellen muss. Im Folgenden sollen nun exemplarisch einige der Einschränkungen von Menschenrechten wie u.a. das Recht auf Freizügigkeit, Art. 13 der Allgemeinen Erklärung der Menschenrechte der UN von 1948 (AEMR1948)), das Recht auf politische Teilhabe, Art. 20 und 21 AEMR und das Recht auf Bildung, Art. 26 AEMR und Art. 28 der UN-Kinderrechtskonvention von 1989 (BMFSFG 1993) für Zuwandernde und Zugewanderte als Folgewirkung des aufenthaltsrechtlichen Sonderstatus der anwesenden Nichtzugehörigkeit dargestellt werden.

\section{Der Status der anwesenden Nichtzugehörigkeit und die Menschenrechte}

„Menschenrechte sind grundlegende Rechte, die jedem Menschen allein auf Grund des Menschseins zukommen. Im Zentrum stehen dabei die gleiche Würde und Freiheit der Menschen und deren Solidarität. Dem Geltungsanspruch nach handelt es sich um angeborene, unveräußerliche, egalitäre, unteilbare und universelle Rechte der Individuen, die insbesondere auf der Seite der staatlichen Gewalt mit besonderen Verpflichtungen einhergehen [...]" (Schulte/Treichler, 2010, S.169). Zentraler Bezugspunkt und Grundlage ist Artikel 1 der Allgemeinen Erklärung der Menschenrechte (AEMR 1948) und des Grundgesetzes (1949) „Die Würde des Menschen ist unantastbar", wonach im Kantschen Sinne der Mensch als Zweck seiner selbst zu betrachten und in seiner körperlichen Integrität, personalen Identität und soziokulturellen Zugehörigkeit zu bejahen ist (vgl. Brumlik 2009).

\section{Recht auf Freizügigkeit}

„Die allgemeine Erklärung der Menschenrechte (United Nations [UN] 1948) kennt ein Recht auf grenzüberschreitende Bewegungsfreiheit: Demnach hat jeder das Recht zu emigrieren, also ein Land zu verlassen - allerdings gibt es kein Recht zu immigrieren, also in ein Land einzuwandern (Artikel 13).“ (Benhabib 2008, S.22)

Während innerhalb der Europäischen Union (EU) die Freizügigkeit für die Angehörigen der mittlerweile 27 EU-Mitgliedsstaaten immer weiter zunimmt, werden die Außengrenzen immer rigider gegen Einwanderungswillige abgeschottet. Selbst das in Art. 14 AEMR festgehaltene Recht auf Asyl wird insbesondere in Deutschland immer wirkungsvoller mithilfe der so genannten Drittstaatenregelung unterlaufen. Denn wer vor der Einreise ein sicheres Drittland durchquerte - und Deutschland ist bekanntlich auf anderem Wege als über Land nicht zu erreichen - wird sofort in dieses „sichere Drittland“ zurückgewiesen. Die 
Möglichkeit und das Recht, Fluchtgründe vorzutragen und damit Asyl zu beantragen, werden auf diese Weise entzogen (vgl. auch Sachverständigenrat 2010, S.69).

Wer nicht Schutz vor Verfolgung sucht, sondern der Armut und dem Hunger in seinem Heimatland entfliehen will, hat ebenso wenig Chancen, einen Aufenthalt hier zu erlangen. Nur bei hoher Qualifikation und über sehr aufwändige bürokratische Wege ist eine Arbeitseinwanderung nach Deutschland noch möglich, wobei nicht einmal gesichert ist, dass die im Ausland erworbenen akademischen und Berufsabschlüsse - trotz großen Fachkräftebedarfs - hier anerkannt werden.

Kein Wunder also, dass Schutzsuchende vielfach untertauchen, um der Abschiebung zu entgehen, und Arbeitssuchende versuchen, ,über die grüne Grenze“ einzureisen. Diese ,Menschen ohne Papiere', die nach Art. 1 der AEMR wie „alle Menschen frei und gleich an Würde und Rechten geboren [sind]“, die „mit Vernunft und Gewissen begabt [sind] und einander im Geiste der Brüderlichkeit begegnen [sollen]", sind in Deutschland weithin rechtlos und sie haben im Gegensatz zu anderen Länder, wie z.B. bisher zu Spanien, praktisch keine Chance auf Legalisierung ihres Aufenthalts.

Neben dem Entzug der Einreisefreizügigkeit gelten auch innerhalb Deutschlands für bestimmte Gruppen sich in Deutschland rechtmäßig aufhaltender Menschen Einschränkungen der Freizügigkeit. Für alle Schutz vor Verfolgung Suchenden, denen es gelingt einen Asylantrag zu stellen, besteht strenge Residenzpflicht. Während des Asylanerkennungsverfahrens sind sie gezwungen, in Gemeinschaftsunterkünften unter vielfach menschenunwürdigen Bedingungen $\mathrm{zu}$ leben ${ }^{1}$. Zudem ist ihnen verboten, Arbeit aufzunehmen, den ihnen zugewiesenen Ort zu verlassen und sie sind gezwungen, ihr Leben von einem um $35-50 \%$ geminderten Sozialhilfesatz zu fristen (vgl. § 3, Asylbewerberleistungsgesetz 1993).

Für die Schutzsuchenden, deren Antrag abgelehnt wurde, die aber nicht in ihr Verfolgerland abgeschoben werden können, gelten dieselben Auflagen bis hin zum völligen Entzug der Bewegungsfreiheit, d. h. in Abschiebehaft genommen zu werden. Diese Auflagen, Beschränkungen, Behinderungen und Ausgrenzungen haben weitere Einschränkungen menschenrechtlicher und grundgesetzlicher Rechte der Person zur Folge: Art. 3 der AEMR und Art.2 des Grundgesetzes $(G G)$ garantieren zwar jedem Menschen das Recht auf Leben, Freiheit, Sicherheit und körperliche Unversehrtheit, doch wird dieses Recht für Nichtdeutsche in $\S 106$ des Aufenthaltgesetzes (AufenthG) explizit eingeschränkt.

Ebenso wenig gilt nach $\S 86$ Aufenth $G$ für Nichtdeutsche der umfassende Datenschutz. Der von Art. $6 G G$ garantierte Schutz von Ehe, Familie und Kindern wird insbesondere bei unsicherem Aufenthalt ${ }^{2}$ und in Fragen der Familienzusammenführung ${ }^{3}$ oft missachtet. Zudem sieht das Grundgesetz selbst wichtige 
Grundrechte (die Versammlungsfreiheit (Art.8), die Vereinigungsfreiheit (Art.9), die Berufsfreiheit und freie Wahl der Ausbildungsstätte) nur als Grundrechte für deutsche Staatsangehörige vor, wiewohl diese Rechte und Freiheiten in der AEMR allen Menschen zustehen.

\section{Verweigerung politischer Teilhabe}

„Das deutsche Recht macht Migranten unsichtbar. Sie sind in der Demokratie nicht repräsentiert.“ Diese Feststellung von Mark Terkessidis (2000, S.73) trifft in vollem Umfang auf die Menschen aus außereuropäischen Staaten, den so genannten Drittstaaten, zu. § 47 AufenthG untersagt allen Drittstaatern die politische Betätigung bzw. verfügt deren Einschränkung, und Art. 28 GG schließt Drittstaater vom Wahlrecht aus, während EU-Bürgerinnen und Bürger seit 1992 zumindest bei Wahlen auf kommunaler Ebene ihre demokratischen Rechte wahrnehmen können. Dies bedeutet, dass knapp 5 Millionen Eingewanderten mit ausländischem Pass, die seit mehr als 5 Jahren in Deutschland dauerhaft und rechtmäßig ansässig sind, das Wahlrecht auf jeder politisch-institutionellen Ebene verwehrt wird. Dazu sei an dieser Stelle die „Einschätzung und Empfehlung von der European Commission against Racism und Intolerance (ECRI) zur Beteiligung von Ausländern am kommunalen öffentlichen Leben in Deutschland“" zitiert (nach Schulte/Treichler 2010, S. 97):

„Keinerlei Schritte wurden [in Deutschland] unternommen, um das Übereinkommen über die Beteiligung von Ausländern am kommunalen öffentlichen Leben zu unterzeichnen. Den Angaben der Behörden zufolge ist auch nicht daran gedacht, dieses Übereinkommen in nächster Zeit zu unterzeichnen (...) ECRI wiederholt die Aufforderung an Deutschland, das Übereinkommen über die Beteiligung von Ausländern am kommunalen öffentlichen Leben zu unterzeichnen und zu ratifizieren (...).“(ECRI 2009, 13f)

Diese gesetzlich und grundrechtlich fixierten Benachteiligungen, Ausgrenzungen und Ungleichbehandlungen insbesondere von Eingewanderten aus Drittstaaten bauen hohe Integrationsschranken auf. Sie stellen erbrachte Integrationsleistungen immer wieder neu in Frage und sie bewirken, wie Terkessidis (2000, S.92) formuliert, dass ,selbst die Nachkommen der ,Gastarbeiter' nicht in der deutschen Gesellschaft ankommen" können.

Ähnlich wie die institutionelle Verweigerung politischer Beteiligung dauerhaft hier lebender Menschen wirken die benachteiligenden, zur sozialen Unterschichtung führenden Regelungen im Arbeitsbereich, durch welche die Arbeit ausländischer Arbeitkräfte als unqualifizierte körperliche Arbeit festgelegt wird. Nicht umsonst wurden ja die zwischen 1955 und 1973 Angeworbenen vor allem auf ihre körperliche Verfassung und Tauglichkeit zu schwerer Arbeit untersucht 
und für niedrig bezahlte körperliche Arbeit, oft wirkliche Schwerstarbeit, eingestellt. Zum anderen drängte die in Deutschland bisher geradezu systematisch betriebene Nicht-Anerkennung von im Herkunftsland erworbenen Qualifikationen die Menschen in Bereiche unqualifizierter Arbeit mit den entsprechenden Niedriglöhnen. Erst jetzt, im Sommer 2011, liegt dem Bundestag ein Gesetzentwurf zur Beratung vor, durch den die Bundesregierung „die Anerkennung von Berufsabschlüssen aus dem Ausland verbessern“ will (Das Parlament, 4. 7. 2011). Es liegt auf der Hand, dass diese institutionellen Rahmenbedingungen erhebliche Auswirkungen auf die soziale Lage der Eingewanderten und auf ihre kulturellen, politischen und schulischen Planungs- und Gestaltungsmöglichkeiten haben.

\section{Das Recht auf Bildung}

Dass die als Konjunkturpuffer und hin und her schiebbare Ware angeworbenen Arbeitskräfte Kinder und Familie haben könnten, war nicht geplant. 1964, immerhin 9 Jahre nach dem ersten Anwerbeabkommen, wurde zwar die Schulpflicht für Kinder aus so genannten ,Gastarbeiterfamilien' von der Kultusministerkonferenz (KMK) verfügt, ihre Umsetzung zog sich jedoch in manchen Bundesländern bis in die 70er Jahre hinein. Für Kinder und Jugendliche aus Flüchtlingsfamilien und aus Familien, deren Eltern lediglich geduldet sind (vgl. Bielefeld 2007), besteht diese Grauzone z.T. sogar bis heute.

Weitestgehend ungesichert ist das Recht auf Bildung für schulpflichtige Kinder und Jugendliche aus Familien ohne ,rechtmäßigen Aufenthaltstitel“. Sie hatten - vom Entgegenkommen mancher Schulleitungen abgesehen - in allen Bundesländern über Jahrzehnte keine Möglichkeit des Schulbesuchs. Das Menschenrecht auf Bildung (Art. 26 AEMR 1948 und Art. 28 der Kinderrechtskonvention 1989), das wie alle Menschenrechte unabhängig von Herkunft und Status gilt, konnte und kann offiziell nicht in Anspruch genommen werden, ohne den Aufenthalt in Deutschland zu gefährden, denn die $\S \S 75$ und 76 AuslG (in Kraft bis 2004) und der $\S 86$ AufenthG (in Kraft seit 2005) verpflichten alle öffentlichen Stellen, alle ihnen bekannt werdenden Daten zum Aufenthalt von Menschen mit ausländischem Pass den Ausländerbehörden zu melden. Eine Studie des Sachverständigenrats ergab, „dass es in Deutschland bis zu 30000 irreguläre Kinder im schulpflichtigen Alter gibt“" (Sachverständigenrat 2010, S.142, Anm.30)

Zwar sah schon der Koalitionsvertrag von Oktober 2009 vor, diese Übermittlungsverpflichtung von staatlichen vorschulischen und schulischen Einrichtungen generell aufzuheben (vgl. Die Zeit, 22.12.2009, Die Phantomschüler), doch erst jetzt, im Sommer 2011, hat der Bundestag beschlossen, „dass Schulen künftig nicht mehr verpflichtet sind, Kinder und Jugendliche, die keine Papiere haben, den Ausländerbehörden zu melden“ (Frankfurter Rundschau, 9./10. 7. 
2011). Einzelne Städte und Bundesländer, so z.B. München, Bremen, Hamburg, Hessen, Nordrhein-Westfalen und Schleswig Holstein ${ }^{4}$, hatten jedoch bereits in den letzten Jahren diese Übermittlungspflicht in unterschiedlichem Ausmaß aufgehoben und so das in der AEMR und im Übereinkommen über die Rechte des Kindes festgeschriebene Recht auf Bildung erfüllt ${ }^{5}$.

\section{Im Labyrinth der deutschen Bildungspolitik}

Die in den 70er Jahren sich verallgemeinernde Beschulung von Kindern und Jugendlichen anderer Kultur- und Spracherfahrungen traf auf völlig unvorbereitete Kollegien in den Schulen, die dann versuchten den für sie unaufwändigsten Weg zu gehen. Kinder im Grundschulalter wurden grundsätzlich ein bis zwei Klassenstufen tiefer eingestuft, meist sogar ins erste Schuljahr:

„Die sind doch alle zwei oder drei Jahre jünger als ich. [...] Ich bin doch erst mit acht in Deutschland in die Schule gekommen, und weil ich die deutsche Sprache nicht kann, musste ich zu den ganz Kleinen.“ (Ude-Pestel 1981, S.49)

Ältere Kinder, auch wenn sie im Herkunftsland das Gymnasium besucht hatten, kamen und kommen grundsätzlich wegen mangelnder Deutschkenntnisse in die Hauptschule. In den 70er Jahren bis weit in die 80er Jahre mussten Seiteneinsteiger oft Nationalitätenklassen besuchen.

Auf eine weitere Form diskriminierender Vorab-Einstufung macht Sineb el Masrar in ihrem Buch Muslim Girls (2010, S.72) aufmerksam:

„Mindestens einmal im Leben passierte es jedem von uns, dass einem ein Lehrer ohne Umschweife sagte, dass eine Drei oder Vier für ein Ausländerkind völlig ausreichend sei. Egal wie gut es um unsere Allgemeinbildung und Schulleistung stand, wir blieben auf den unteren Rängen der Bewertungsskala hängen. Andere Lehrer befanden schlichtweg: ,Ich lasse euch schon durchfallen!““

Hinzu kam und kommt die unter Lehrern verbreitete Fehleinschätzung, dass schon allein durch Zuhören und Dabeisein die Schülerinnen und Schüler aus Einwandererfamilien schon Deutsch lernen würden. Kamen sie nicht mit, mussten sie die Klasse wiederholen oder kamen in die Sonderschule für Lernhilfe. Noch immer wiederholen Grundschüler und -schülerinnen mit sog. Migrationshintergrund viermal so oft eine Klasse wie ihre Klassenkameraden und -kameradinnen ohne Migrationshintergund (vgl. Konsortium Bildungsberichterstattung 2006, S.152). Zwar gibt es in manchen Bundesländern seit ca. 20 Jahren Intensivkurse in Deutsch als Zweitsprache, aber - wie immer - in viel zu geringem 
Umfang und mit viel zu wenigen dafür ausgebildeten Lehrerinnen und Lehrern. Dass Deutsch als Zweitsprache ganz anders unterrichtet werden muss als Deutsch als Muttersprache. trat erst nach und nach in das didaktische Bewusstsein ein.

Erst seit der Veröffentlichung der PISA-Ergebnisse wurden in manchen Bundesländern, wie z.B. in Hessen, flächendeckend Vorlaufkurse in Deutsch als Zweitsprache eingerichtet. Doch bis heute sind viele Lehrerinnen und Lehrer noch immer der Meinung, dass eine Sprache, in deren Land man lebt, so nebenbei und natürlich gelernt wird. Auf diesem Weg kann durchaus umgangssprachliche Kompetenz erworben werden, nicht aber die situationsunabhängige, abstrahierende Sprache, in der Lernen in der Schule stattfinden muss. Die Bi- und Plurilingualität der Schülerinnen und Schüler wiederum erfährt kaum je Würdigung. Sie gilt als Problem ${ }^{6}$.

Unter diesen Umständen ist kaum überraschend, dass die zweite Generation der Migranten, die mittlerweile die Eltern- und Großeltern der gegenwärtigen Schülerinnen und Schüler sind, schon früh als eine schulisch und sozial verlorene Generation galt. „Der integrierende Effekt der Schulbildung [bleibt] für ausländische Kinder und Jugendliche bisher weitgehend wirkungslos," schrieb schon H. Kühn in seinem Memorandum (1979, S.24)7. Die PISA-Ergebnisse zeigen, dass sich strukturell bis heute wenig geändert hat. Noch immer sind - auch wenn sich inzwischen durchaus positive Veränderungen zeigen (Sachverständigenrat 2010, S.137ff) - Migrantenkinder und -jugendliche weithin ins Bildungsabseits gedrängt. Die Leugnung des Einwanderungstatbestands hat bis heute verhindert, Schulbildung auf die Erfordernisse eines Einwanderungslandes umzustellen.

Trotz dieser faktisch eher auf Exklusion, die Aufrechterhaltung eines Sonderstatus der anwesenden Nichtzugehörigkeit statt auf Inklusion zielenden Regelungen, und der häufig ebenso exkludierenden Praxis in Schule und Unterricht gelang es einer zunehmenden, jedoch immer noch zu geringen Zahl von Schülerinnen und Schülern aus Einwandererfamilien der 2. und 3. Generation, bemerkenswerte Bildungserfolge zu erzielen. Diese sind allerdings, wie in autobiographischen Berichten oder Beratungsgesprächen deutlich wird, oft dem glücklichen Zufall eines aufmerksamen persönlichen Umfeldes zu verdanken. So erzählt die freie Journalistin und Autorin Hatice Akyün (2011, S.219), dass sie ihre Erfolge

„den Müttern meiner Schulfreundinnen zu verdanken“ habe, „die mir bei den Hausaufgaben geholfen haben. Ich habe sie meiner Lehrerin zu verdanken, die mich in der Schule gefördert hat. Ich habe es also in gewisser Weise dem Zufall zu verdanken, dass ich engagierten Menschen begegnet bin. Wer aber sollte es verantworten, dass die Zukunft von Migrantenkindern einem Glücksspiel gleicht?“”

Sineb El Masrar hebt hervor (2010, S.71f), dass der Erfolg vieler Schülerinnen und Schüler ,ausschließlich dem lernfreundlichen Umfeld“", das ihre Eltern ihnen 
schufen, aber „,vor allem dem Glück, auf fördernde Lehrer gestoßen“ zu sein, zu verdanken sei.

In der von Widersprüchen hoch geladenen Bildungsrealität stand die Schulpädagogik in den 70er/80er Jahren vor dem Dilemma, gesellschaftlich bedingte Missstände pädagogisch abfangen zu sollen, ohne dass die Schulpolitik die entsprechenden Rahmenbedingungen geschaffen hätte. „Klassisch“, im deutschen Schulwesen sozusagen pfadbedingt, war der meist gewählte Lösungsansatz: nämlich die im monokulturellen, auf Nationalbildung ausgerichteten Schulsystem - aus eben dieser Sicht - Probleme verursachenden Schülerinnen und Schüler zu Zielgruppen sonderpädagogischer Überlegungen zu machen. Statt den Blick auf Struktur und Konzeption von Schule zu lenken, statt zu fragen, ob Schule noch die angemessenen Antworten auf die Anforderungen einer veränderten Schülerpopulation hat, wurden auch im pädagogischen Diskurs zunächst vor allem kompensatorische Maßnahmen diskutiert und ersonnen - die Ausländerpädagogik. Franz Hamburger (2009) argumentierte bereits seit Beginn der 80er Jahre gegen diese sonderpädagogische Blickweise ebenso wie später gegen den kulturzentrierten Ansatz der ,Interkulturellen Erziehung ‘. Er prägte früh den Begriff der Pädagogik der Einwanderungsgesellschaft, der gesamtgesellschaftliche Aspekte in den pädagogischen Diskurs einbezieht.

Die Wahrnehmung der Eingewanderten und ihrer Kinder als „Problem“, als „Träger von Defiziten“ blieb lange für die Problem- und Theoriebearbeitung der Pädagogik im Einwanderungsland bestimmend (vgl. Gomolla / Radtke 2000) und ist - siehe auch die Thesen von Thilo Sarrazin und die Debatte darum - immer noch in der öffentlichen Debatte kampagnenmächtig und durchschlagskräftig (vgl. G. Steffens in diesem Band).

Dies schlägt sich auch in den Mikrostrukturen der Alltagskommunikation und Einzelentscheidungen nieder: Besucht eine größere Zahl von Kindern aus Einwandererfamilien, deren Migration ja vielfach schon mehrere Jahrzehnte zurückliegt, eine Klasse oder eine Schule, so führt oft schon der Umstand der ausländischen Namen, einer mit dem Namen unterstellten anderen Kultur und einer anderen in der Familie gesprochenen Sprache zu einer Wahrnehmungsweise, aus der von schwierigen pädagogischen, ja sonderpädagogischen Bedingungen gesprochen wird (K. Steffens 2006, S.396). Der Defizitargumentation dienen als Maßstab die monokulturell und mittelschichtzentriert gesetzten Bildungsnormen, um festzustellen, was ausländischen Schülerinnen und Schülern an Kenntnissen und Vorbildung fehlt. Es lässt sich leicht nachvollziehen, dass die betreffenden Schülerinnen und Schüler, über deren besondere Sprachkompetenzen dabei hinweg gesehen wird, dies als deprimierende, deklassierende Botschaft wahrnehmen müssen. 
Obwohl schon Ende der 60er Jahre der Zusammenhang von Schichtzugehörigkeit und Schulerfolg breit erörtert worden war (Friedeburg 1986), hatte diese Diskussion kaum Eingang in die Debatte um die pädagogische Arbeit mit Kindern aus Einwandererfamilien gefunden - ein umso stärkeres Versäumnis, als gerade die Auswirkung von sozialer Unterschichtung über die oben schon angesprochene Anwerbe- und Arbeitsmarktpolitik der Bundesregierung auf die Bildungschancen von Migrantenkindern und -jugendlichen massiv war. Schon die Anwerbepolitik bis 1973 war ja auf Arbeitskräfte für schwere, schlecht bezahlte und oft gesundheitsgefährdende Arbeit fokussiert, die in der Regel keine Ausbildung erforderte und für die sich keine Einheimischen fanden. Diese arbeitsmarktpolitische Leitvorstellung galt dann weiterhin für alle Einwandernden, seien es nachziehende Familienangehörige oder - seit den 80er Jahren - auch Flüchtlinge, die bis zum vergangenen Jahr 2010 trotz oft hoher beruflicher Qualifikationen gemäß der Vorrangigkeitsklausel (Arbeitsplätze müssen zuerst deutschen Arbeitssuchenden angeboten werden) nur zu solchen minderen Arbeitsplätzen Zugang erhielten. Diese Anwerbe- und Arbeitsmarktpolitik hat zwangsläufig zu einer Unterschichtung der deutschen Gesellschaft durch Eingewanderte geführt, weil sie selbst Eingewanderte mit hoher Schul- und Berufsbildung für die dem entsprechenden beruflichen Bereiche nicht zuließ. Über Jahrzehnte wurde nicht reflektiert, dass die Anwerbung gering qualifiziert eingestufter Arbeitskräfte und die fortgesetzte staatlich organisierte soziale Zuweisung von Eingewanderten auch Auswirkungen auf die Bildungsvoraussetzungen der Kinder und Jugendlichen aus Einwandererfamilien haben musste.

Erst der Bericht der Beauftragten der Bundesregierung für Ausländerfragen (heute: Bundesbeauftragte für Migration, Integration und Flüchtlinge) von 2002 über die Lage der Ausländer benennt diesen Zusammenhang ohne Umschweife:

„Der Schulerfolg wird - weit vor der Einschulung - entscheidend beeinflusst durch das
Anregungspotential und den Erziehungsstil der Eltern. Im Elternhaus werden die Weichen
gestellt für Bildungsmotivation und Lernselbständigkeit. Entsprechende Erziehungsziele
sind in der sozialen Mittel- und Oberschicht jedoch weiter verbreitet als in der sozialen
Unterschicht. Da Kinder und Jugendliche ausländischer Herkunft überproportional häufig
aus Unterschichtfamilien kommen, sind sie in zweifacher Hinsicht strukturell benachtei-
ligt, da sich hier ggf. migrationsspezifische und schichtspezifische Probleme addieren.“
(Bericht 2002, S.174)

Wie in der dominanten öffentlichen Debatte wird auch in der pädagogischen Praxis jedoch die Problemlage nach wie vor meist nicht als sozial, sondern als ethnokulturell verursacht gedeutet. Daher bestehen die sich aus dem Muster der Defizitwahrnehmung ergebenden Probleme weithin fort, weil der Blick auf Ressourcen und Chancen, die etwa Sprachenvielfalt und Sprachkompetenzen bieten, 
blockiert bleibt. Daran haben auch einige erfolgreiche bilinguale Modellprojekte nichts ändern können. Diese beispielhaften Projekte (z.B. Nehr et al., 1988) knüpfen grundsätzlich an den Kenntnissen und Erfahrungen der Schülerinnen und Schüler an. Allerdings wurden und werden solche Modellprojekte gar zu oft finanziell und personell wieder ausgetrocknet, so dass die Erfahrungen nicht verallgemeinert werden konnten. Überdies wurde in den ersten Jahrzehnten nach 1970 die Förderung migrantischer Schulkinder fast vollständig in die außerschulische Bildung ausgelagert. Wohlfahrtsverbände, Initiativen und Ehrenamtliche nahmen sich der so genannten außerschulischen Förderung etwa durch organisierte Hausaufgabenhilfe an. Auch diese Auslagerung trug dazu bei, dass Schule und Kollegien sich zum Überdenken ihrer Konzepte nicht genötigt sahen. Für Schulpolitik und pädagogische Praxis kann daher durchaus verallgemeinernd gesagt werden: Die monokulturelle Bildung als Nationalbildung wurde nicht in Frage und der Fortbestand ihrer Geltung in einer veränderten Welt und Gesellschaft nicht zur Disposition gestellt.

Die unterlassenen Bildungsanstrengungen des Einwanderungslandes Deutschland und die vielfach darauf beruhende soziale Not und Perspektivlosigkeit der Eingewanderten hat eine in Teilen widerständige und widerwillige Schülergeneration aus Einwandererfamilien heranwachsen lassen. Und noch immer zeigen sich große Teile der Lehrerschaft und Schulbehörden unvorbereitet. Immer noch mangelt es an systematischer und umfassender Förderung in Deutsch als Zweitsprache. Zwar wird mit dem Projekt FÖRMIG an ausgewählten Schulen diskutiert und erprobt, dass Deutsch in allen Schulfächern in enger Verbindung mit der fachspezifischen Bildung Gegenstand fachspezifischer Sprachübungen sein sollte. Doch von flächendeckender Praxis dieser Sprachförderung in allen Fächern ist bisher nicht die Rede ${ }^{8}$.

Bildung in einer Einwanderungsgesellschaft wird solange auf kulturalistischen und sonderpädagogischen Nebenpfaden wandeln, solange Gesellschaft und Politik über ihre Protagonisten, Institutionen, Gesetze und Medien kulturelle Fremdheit und soziale Ausgrenzung produzieren und Verwirklichungs- und Lebenschancen von Eingewanderten und ihren Nachkommen beschneiden. Die kulturalistischen, ja rassistischen Denkfiguren führen zu der - erwünschten Verkehrung von Voraussetzung und Folge, nach welcher nicht der für Eingewanderte soziokulturell und sozioökonomisch vorgegebene Raum für das Scheitern $\mathrm{zu}$ vieler Kinder im Bildungsprozess verantwortlich ist, sondern die Eingewanderten selbst. Erst wenn die Umkehr dieser Verantwortungszuschreibung gelänge, könnte sich der Weg für eine Bildung zu Autonomie als universeller und ungeteilter menschenrechtlicher Anspruch eröffnen. Für diese Entwicklung könnten die Vorschläge von Sineb el Masrar Anregungen für einen Bildungsdiskurs im Einwanderungsland bieten: 
„Gefragt sind fortschrittlich denkende Lehrer, die mehr auf eigenverantwortliches Lernen setzen und die Kinder gemäß ihren Fähigkeiten fördern, egal welcher Herkunft." (Sineb el Masrar 2010, S. 69)

„Auch die Abschaffung des dreigliedrigen Schulsystems würde das zukünftige soziale Klima beflügeln, da beim gemeinsamen Lernen unterschiedliche Lebensrealitäten aufeinandertreffen können und die Kinder länger miteinander im Umgang stehen. Gleichzeitig spornen sie sich an und lernen einen respektvolleren Umgang, ohne dass dabei die leistungsstärkeren Kinder unterfordert werden müssen“" (ebd.)

„Doch besonders notwendig sind dabei in erster Linie die Inhalte und die Durchführung der Unterrichtsstunden; solange man diese nicht überdenkt, wird sich an der Qualität der Bildung nichts ändern“ (ebd.) „Es ist höchste Zeit, dass die Lehrpläne in Deutschland aufgefrischt werden (...). Man könnte im Deutschunterricht zeitgenössische Schriftsteller lesen wie Feridun Zaimoğlu“ (ebd. S.79).

„Platz da, jetzt stehen auch wir am Lehrerpult! ... Als Lehrer leben Frau Yilmaz, Frau Lopez, Herr Reza und Herr Aziz zugleich mit großer Selbstverständlichkeit allen Kindern und Eltern - auch den deutsch-deutschen - im Klassenzimmer ein Stück deutsche Lebensrealität vor" (ebd. S.92).

\section{Anmerkungen}

1 vgl. etwa die im Aufruf von ProAsyl gegen die soziale Entrechtung von Flüchtlingen, Faltblatt März 2011, geschilderten Fälle und Verhältnisse

2 vgl. den Fall der Familie Khateeb von Dietzenbach (Frankfurter Rundschau (FR), 7.10.2010) oder des 16-jährigen Schülers Jamal H. (FR, 27.1.2010)

3 vgl. die ausländerrechtlichen Verschärfungen des Nachzugs von Ehepartnern/innen: Verlängerung der Wartefristen, schriftlicher Nachweis von Deutschkenntnissen vor der Einreise (vgl. FR 11.3.2010)

4 vgl. das Hamburgische Schulgesetz von 1997, zuletzt geändert in 2008, §§ 1,2 und 37: „Wer in Hamburg seine Wohnung hat ist zum Schulbesuch verpflichtet“ (Diakonie Hamburg, Leben ohne Papiere, eine empirische Studie, Hamburg 2009, S.147), Hessen z.B. hob diese Übermittlungspflicht im Dezember 2009 auf, vgl. Verordnung zum Schulbesuch, Änderung veröffentlicht im Amtsblatt 12/09, S.850, 15.Dez.2009, vgl. Alt, Fodor, Rechtlos? Menschen ohne Papiere, 2001, S.182ff

5 Die 1992 angefügte Vorbehaltsklausel eingewanderte Kinder betreffend wurde im Mai 2010 aufgehoben. Absatz 1 des Artikels 28 lautet: „Die Vertragsstaaten erkennen das Recht des Kindes auf Bildung an; um die Verwirklichung des Rechts auf der Grundlage der Chancengleichheit fortschreitend $\mathrm{zu}$ erreichen, werden sie insbesondere a) den Besuch der Grundschule für alle zur Pflicht und unentgeltlich machen; b) die Entwicklung verschiedener Formen der weiterführenden Schulen allgemein bildender und berufsbildender Art fördern, sie allen Kindern verfügbar und zugänglich machen und geeignete Maßnahmen wie die Einführung der Unentgeltlichkeit und die Bereitstellung finanzieller Unterstützung bei Bedürftigkeit treffen; (...)“

6 Erfahrungen von institutioneller Diskriminierung insbesondere in der Grundschule tun das Ihre, um Lernlust und Zukunftsoffenheit abzuwürgen. Lehrerinnen und Lehrer an Grundschulen erteilen Kindern mit Migrationshintergrund bei gleicher Leistung im Verhältnis von 1,66 schlechtere Noten als Kindern ohne Migrationshintergrund. 
Ebenso restringierend wird bei der Schulbesuchsempfehlung in die Sekundarstufe I verfahren. Würden Eltern sich nicht über diese Empfehlung, dort wo es möglich ist, hinwegsetzen, wäre der Anteil der Migrantenkinder in höher qualifizierenden Schulzweigen noch niedriger. Konsortium Bildungsberichterstattung, 2006, S.165f.

7 vgl. auch Gomolla, Ratke, Institutionelle Diskriminierung, Opladen 2000. - „Ausländische Jugendliche beenden Schule als halbe Analphabeten“, titelte die FR 1987 und nicht nur sie. Berichte über die schulische Erfolglosigkeit zu vieler Migrantenkinder und -jugendlicher reißen bis heute nicht ab. Auch wenn die Quote der Schulabgänger und -gängerinnen ohne Abschluss von etwa 50\% im Jahr 1979 auf rund $20 \%$ gesunken ist, so ist dies nach so vielen Jahrzehnten ein bitteres Armutszeugnis.

8 FÖRMIG $=$ Förderung von Kindern und Jugendlichen mit Migrationshintergrund, „durchgängige Sprachförderung“ als Gesamtkonzept sprachlicher Bildung, Handreichung, Universität Hamburg, 2006

\section{Literatur}

Akyün, Hatice, 2011, Was ist Heimat, in: Sezgin, Hilal (Hrsg), 2011, S. 213 - 220

Allgemeine Erklärung der Menschenrechte 1948, AEMR 1948, in: Hessische Landeszentrale für politische Bildung (Hrsg.), Verfassungstexte, Wiesbaden 2007, S. $114-119$

Alt, Jörg, Fodor, Ralf, 2001, Rechtlos? Menschen ohne Papiere, Karlsruhe

Asylbewerberleistungsgesetz (AsylbLG) v. 30.6.1993 i.d.F. der Bekanntmachung v.5.8.1997, zuletzt geändert 24.9.2008, in: Ausländerrecht, 2010, S. 452 - 460

Aufenthaltsgesetz (AufenthG) v. 30.7.2004 i.d.F. der Bekanntmachung v.25.2.2008, zuletzt geändert 30.7.2009, in: Ausländerrecht, 2010, S. 1 - 97

Ausländerrecht, 2010, Textausgabe mit ausführlichem Sachverzeichnis und einer Einführung von Dr. Klaus Dienelt, 24., überarbeitete Auflage, Stand: 3. März 2010, München

Benhabib, Seyla, 2008, Die Rechte der anderen, Frankfurt a.M.

Bericht 2002, Bericht der Beauftragten der Bundesregierung für Ausländerfragen über die Lage der Ausländer in der Bundesrepublik Deutschland, Berlin und Bonn, September 2002

Bielefeld, Heiner, 2007, Menschenrechte in der Einwanderungsgesellschaft, Bielefeld

BMFSFG (Hrsg.), 1993, Übereinkommen über die Rechte des Kindes. UN-Konvention im

Wortlaut mit Materialien. Texte in amtlicher Übersetzung. Bundesministerium für Familie, Senioren, Frauen und Gesundheit: Bonn.

Brumlik, Micha, 2009, „Leitkultur“: Würde des Menschen, in: Erziehung und Wissenschaft $2 / 2009$

Diakonie Hamburg, 2009, Leben ohne Papiere, eine empirische Studie zur Lebenssituation von Menschen ohne gültige Aufenthaltspapiere in Hamburg, Hamburg

El Masrar, Sineb, 2010, Muslim Girls - Wer wir sind, wie wir leben, Frankfurt

Friedeburg, Ludwig v., 1989, Bildungsreform in Deutschland. Geschichte und gesellschaftlicher Widerspruch, Frankfurt

Hamburger, Franz, 2009, Abschied von der Interkulturellen Pädagogik, Weinheim

Heckmann, Friedrich, 1992, Ethnische Minderheiten, Volk und Nation, Stuttgart

Keim, Wolfgang, Steffens, Gerd, 2006, Bildung und gesellschaftlicher Widerspruch, Frankfurt 
Konsortium Bildungsberichterstattung (Hrsg.), 2006, Bildung in Deutschland, Bielefeld Kühn, Heinz, Memorandum, 1979, Stand und Perspektiven der Integration

Nehr, Monika et al., 1988, In zwei Sprachen lesen lernen - geht denn das?, Weinheim und Basel

Sachverständigenrat deutscher Stiftungen für Integration und Migration (SVR), 2010, Einwanderungsgesellschaft 2010 - Jahresgutachten, Berlin

Schulte, Axel; Treichler, Andreas, 2010, Integration und Antidiskriminierung, Weinheim Sezgin, Hilal (Hrsg.), 2011, Manifest der Vielen, Deutschland erfindet sich neu, Berlin

Sezgin, Hilal, 2011, Deutschland schafft mich ab, in: Sezgin, Hilal (Hrsg.), 2011, S. 45 52

Steffens, Karin, 2006, in: Keim, Wolfgang, Steffens, Gerd, 2006, S. $389-410$

Terkessidis, Mark, 2000, Migranten, Rotbuch 3000, Hamburg

Ude-Pestel, Anneliese, 1981, Ahmet, - Geschichte einer Kinder-Therapie, München 